\title{
Efectos de heterosis en poblaciones nativas de maíz y sus cruzas $F_{1}$
}

\author{
Effects of heterosis on native populations of maize and their $F_{1}$ crosses
}

\begin{abstract}
Yessica F. Cervantes-Adame1*, Hermes Rebolloza-Hernández'1, Elizabeth Broa-Rojas², Angeluz Olvera-Velona', Gregorio Bahena-Delgado'

1 Escuela de Estudios Superiores de Xalostoc, Universidad Autónoma del Estado de Morelos (UAEM), Av. Nicolás Bravo S/N, Parque Industrial Cuautla, Ayala, Morelos. CP. 62715.Tel. (777) 3297000 Ext. 7981.

2 Escuela de Estudios Superiores de Totolapan, Universidad Autónoma del Estado de Morelos (UAEM) Carretera TotolapanNepopualco S/N Ahuatlán Totolapan Morelos CP.62830, Tel. (735) 3579977.
\end{abstract}

\section{RESUMEN}

Con el objetivo de cuantificar la Heterosis promedio (Hp) y determinar los efectos de Heterosis varietal $\left(h_{j}\right)$ y Heterosis específica $\left(s_{\mathrm{jij}}\right)$, se utilizaron siete poblaciones progenitoras de maíz nativo y sus 21 cruzas directas. Los 28 genotipos de maíz se evaluaron en tres ambientes del estado de Morelos (Ayala-O/I-2012/2013, Ayala-P/V-2013 y Tepalcingo-P/V-2013). El diseño experimental fue bloques completos al azar con tres repeticiones. Se midieron cuatro variables y se sometieron a un análisis de varianza combinado, comparación de medias $\left(\mathrm{DMS}_{0.01}\right)$, la Hp se calculó de manera porcentual, la Heterosis varietal y Heterosis específica se determinaron por el método de Gardner y Eberhart. En la expresión porcentual la Cruza-17 presentó valores positivos en $\mathrm{Hp}$ en las variables de GM (19.16), HM (11.81), P100G (7.77) y RG (5.36). En la ( $h_{j}$ ) los progenitores con mayor efecto fueron BCP1 y CB029 ambos impactaron en la formación de cruzas con los valores más altos de $\mathrm{s}_{\mathrm{j} j}$ como la Cruza-26 y Cruza-56 esto para RG y GM. Lo anterior permite la selección de poblaciones nativas de maíz para mejorar características relacionadas al rendimiento de grano mediante el cruzamiento inter-poblacional.

Palabras clave: efecto varietal; cruzas; rendimiento

\section{ABSTRACT}

With the aim of quantifying the average Heterosis $(\mathrm{Hp})$ and determine the effects of varietal heterosis $\left(h_{j}\right)$ and specific heterosis $\left(s_{\mathrm{jij}}\right)$ seven progenitor populations of native maize were used and their 21 direct crosses. The 28 maize genotypes were evaluated in three environments in the state of Morelos (Ayala-O/l-2012/2013, Ayala-P/V-2013 and Tepalcingo-P/V-2013). The experimental design, were complete blocks at random with three repetitions. Four variables were measured and subjected to a combined variance analysis, mean comparison $\left(\mathrm{DMS}_{0.01}\right), \mathrm{Hp}$ was calculated as a percentage, and the varietal heterosis and specific heterosis were determined by the Gardner and Eberhart method. In the percentage expression the Cross-17 presented positive values in $\mathrm{Hp}$ in the variables of GM (19.16), HM (11.81), P100G (7.77) and RG (5.36). In the (h) the progenitors with greater varietal effect were BCP1 and $\mathrm{CB} 029$, both impacted in the formation of crosses with the highest $\mathrm{s}_{\mathrm{jij}}$, values as the Cross-26

*Autor para correspondencia:Yessica Flor Cervantes Adame

Correo electrónico: yessica.cervantes@uaem.mx

Recibido: 15 de julio de 2019

Aceptado: 17 de enero de 2020 and Cross-56 this for RG and GM. This allows the selection of native maize populations to improve characteristics related to grain yield through inter-population crossing.

Keywords: varietal effect; crosses; yield

\section{INTRODUCCIÓN}

La amplia diversidad genética en el maíz, se encuentra representada por la gran cantidad de poblaciones nativas de maíz (Ángeles et al., 2010). La evolución de este germoplasma ha llevado a la formación de las 59 razas de maíz, mismas que han mostrado características agronómicas sobresalientes (Gaytán y Meyec, 2010). El cruzamiento de poblaciones nativas de maíz con diferente grado de divergencia genética (Romero et al., 2002) podría generar cruzamientos interpoblacionales que presenten potencial de rendimiento de grano superior al de sus progenitores (Vergara et al., 2005). La amplia diversidad presente en el maíz nativo, es pieza fundamental en los programas de mejoramiento genético (Esquivel et al., 2009), diversas investigaciones se han enfocado a mejorar características de interés agronómico (Soengas et al., 2003). Por consiguiente, es necesario valorar y desarrollar estrategias que permitan determinar los efectos genéticos mediante el comportamiento heterótico, esto con la finalidad de identificar combinaciones superiores y así, poder seleccionar los mejores progenitores de maíz nativo para el diseño de estrategias de mejoramiento eficientes (Gutiérrez et al., 2002; Preciado et al., 2005).

En la actualidad la heterosis representa la base en el mejoramiento genético por hibridación (Dzib et al., 2011). Los diferentes efectos se manifiestan como la diferencia en vigor entre un híbrido $F_{1}$ y el promedio de ambos progenitores (Hoecker et al., 2006). Si la diferencia en el comportamiento inter-poblacional, proviene de progenitores nativos no emparentados con amplia diversidad genética, puede asegurarse la existencia de heterosis (De la Cruz et al., 2010; Moll et al., 1962). El efecto de heterosis se ha utilizado en diversas investigaciones enfocadas a potencializar el germoplasma nativo de maíz existente (Cress, 1966).

Los maíces nativos, al presentar una amplia diversidad genética son una fuente natural de combinaciones alélicas, esta base germoplásmica puede ser utilizada para identificar progenitores con efectos genéticos sobresalientes y obtener 
cruzas con patrones heteróticos significativos (Gardner and Eberhart, 1966; González et al., 2016). Lo anterior se puede lograr gracias a la interacción alélica que contribuye a la predicción que tendrá cada uno de los progenitores con su respectiva progenie (Esquivel et al., 2011). Por lo tanto, es importante determinar los efectos de heterosis varietal y especifica mediante el análisis de los parámetros genéticos de Aptitud combinatoria general (ACG) y Aptitud combinatoria especifica (ACE) para identificar progenitores y cruzas inter-poblacionales con indicadores sobresalientes de interés agronómico en el rendimiento y sus componentes. (Preciado et al., 2005; Dzib et al., 2011).

Los diferentes efectos atribuidos a factores genéticos de tipo aditivo y no aditivo (dominancia y sobredominancia), efectos epistáticos y la interacción genotipo por ambiente (Griffing, 1956; Falconer, 1996), pueden ser analizados mediante el uso de los diseños dialélicos, mismos que permiten estimar los componentes de la variación genética y los diferentes efectos genéticos (Maldonado et al., 2009), esto con la finalidad de identificar germoplasma sobresaliente con potencial heterótico (Nájera et al., 2010; Han et al., 1991).

Con base en la importancia del maíz nativo en la agricultura, se hace necesaria su evaluación para conocer y determinar su valor agronómico con la finalidad de generar programas de mejoramiento específicos con fines particulares. En este contexto los objetivos de la presente investigación fueron: Estimar el grado de Heterosis promedio en rendimiento y componentes del rendimiento y determinar los efectos de Heterosis varietal $\left(h_{\mathrm{j}}\right)$ y específica $\left(\mathrm{s}_{\mathrm{j} j}\right)$ en siete poblaciones progenitoras de maíz nativo y sus 21 cruzas directas.

\section{MATERIALES Y MÉTODOS Material genético}

El germoplasma utilizado en la presente investigación fueron siete poblaciones nativas de maíz (Tabla 1), colectadas y mantenidas por el Programa de Mejoramiento Genético de Maíz de la Escuela de Estudios Superiores de Xalostoc, estas se seleccionaron previamente como germoplasma progenitor por ser de distinto origen geográfico y por presentar características sobresalientes en rendimiento de grano en experimentos previos de temporal y riego. Tres de las siete poblaciones progenitoras son del estado de Morelos BJM1, BJM2 colectadas en la localidad de Jonacatepec, presentan características afines a la raza Tuxpeño Norteño, CB029 colectada en Yautepec con características afín a Tabloncillo; tres poblaciones del estado de Tamaulipas, Caballeros y Moreño colectadas en la localidad de Padilla con afinidad a la raza Tuxpeño Norteño y la población Ratón colectada en Victoria, esta pertenece a la raza Ratón; y una población proveniente del estado de Puebla BCP1 colectada en la localidad de Coacalco y presenta una mezcla de razas tales como Ratón y Cristalinos del Caribe. Así mismo se evaluaron 21 cruzas formadas a partir de los progenitores nativos antes descritos, dando un total de 28 poblaciones.
Tabla 1. Origen del germoplasma evaluado en el estado de Morelos. Table 1. Origin of germplasm evaluated in the state of Morelos.

\begin{tabular}{|c|c|c|c|c|}
\hline Progenitores & $\begin{array}{c}\text { Origen } \\
\text { Geográfico }\end{array}$ & Raza & $\begin{array}{c}\text { Tipo } \\
\text { de clima }\end{array}$ & $\begin{array}{l}\text { Altitud } \\
\text { (m) }\end{array}$ \\
\hline CB029 & Yautepec, Mor & Tabloncillo & $\begin{array}{c}\text { Cálido- } \\
\text { subhúmedo }\end{array}$ & 1210 \\
\hline BJM1 & $\begin{array}{c}\text { Jonacatepec, } \\
\text { Mor. }\end{array}$ & $\begin{array}{l}\text { Tuxpeño- } \\
\text { Norteño }\end{array}$ & $\begin{array}{l}\text { Cálido- } \\
\text { subhúmedo }\end{array}$ & 1290 \\
\hline BJM2 & $\begin{array}{c}\text { Jonacatepec, } \\
\text { Mor }\end{array}$ & $\begin{array}{l}\text { Tuxpeño- } \\
\text { Norteño }\end{array}$ & $\begin{array}{l}\text { Cálido- } \\
\text { subhúmedo }\end{array}$ & 1290 \\
\hline Ratón & Victoria, Tam. & Ratón & Cálido-seco & 400 \\
\hline Moreño & Padilla, Tam. & $\begin{array}{l}\text { Tuxpeño- } \\
\text { Norteño }\end{array}$ & Cálido-seco & 400 \\
\hline Caballeros & Padilla, Tam. & $\begin{array}{l}\text { Tuxpeño- } \\
\text { Norteño }\end{array}$ & Cálido-seco & 320 \\
\hline $\mathrm{BCP} 1$ & $\begin{array}{l}\text { Coacalco, } \\
\text { Pueb. }\end{array}$ & $\begin{array}{c}\text { Ratón y } \\
\text { cristalinos } \\
\text { del Caribe) }\end{array}$ & Trópico-seco & 698 \\
\hline
\end{tabular}

El incremento de las poblaciones que sirvieron como progenitoras y la formación de las cruzas, se realizó en el ciclo agrícola primavera-verano con fecha de siembra de 19 de junio 2012 bajo condiciones de temporal, en el campo experimental de la Escuela de Estudios Superiores de Xalostoc (Localidad Ayala, Morelos), la parcela experimental presenta un suelo tipo vertisol y se localiza a $18^{\circ} 43^{\prime}$ (latitud norte), $98^{\circ}$ 54' (longitud oeste) con una altitud de $1300 \mathrm{msnm}$; muestra temperatura y precipitación promedio anual de $24^{\circ} \mathrm{C}$ y 992 $\mathrm{mm}$ respectivamente.

\section{Ubicación de la evaluación experimental de progenitores y cruzas $F_{1}$ de maíz nativo}

La evaluación agronómica de los siete progenitores nativos de maíz y sus 21 cruzas directas, se llevó a cabo mediante experimentos de campo conducidos en tres ambientes; (AMB-1) definido por la localidad de Ayala, Morelos en el ciclo agrícola de otoño-invierno 2012/2013 bajo condiciones de riego (AMB-2) localidad de Ayala, Morelos en el ciclo de primavera-verano 2013 bajo condiciones de temporal. Las dos evaluaciones se realizaron en el campo experimental de la Escuela de Estudios Superiores de Xalostoc, de la Universidad Autónoma del Estado de Morelos, la parcela presenta las mismas características descritas anteriormente; (AMB-3) se estableció en el ciclo primavera-verano 2013 bajo condiciones de temporal en la localidad de Tepalcingo, Morelos. La parcela agrícola denominada Campo las pilas, presenta un tipo de suelo arcilloso y se localiza a $18^{\circ} 35^{\circ}$ (latitud norte), $98^{\circ} 50^{\prime}$ (longitud oeste) con una altitud de $1180 \mathrm{msnm}$; muestra temperatura y precipitación promedio de $24,3^{\circ} \mathrm{C}$ y $885,3 \mathrm{~mm}$ respectivamente.

\section{Conducción de experimentos}

La siembra de las evaluaciones se realizó el 3 de febrero 2013 (AMB-1), 29 de junio2013 (AMB-2) y 2 de julio 2013 (AMB-3). La siembra se llevó a cabo de forma manual, depositando dos semillas cada $0.25 \mathrm{~m}$ con una distancia entre surcos de $0.80 \mathrm{~m}$. Se realizó un aclareo cuando las plantas 
presentaron la quinta y sexta hoja, dejando una planta por mata. La fertilización se realizó con las dosis 160-70-00 (ciclo otoño-invierno) y 160-70-60 (ciclo primavera-verano) en ambos casos se aplicaron 100 ppm de nitrógeno y la totalidad del fosforo al momento de la siembra, el resto de nitrógeno se distribuyó al realizar la segunda escarda. El control de maleza e insectos se llevó a cabo de acuerdo a lo recomendado para la producción de maíz en el Estado de Morelos.

\section{Diseño y unidad experimental}

Para la evaluación de las 28 poblaciones, se utilizó un diseño experimental bloques completos al azar con tres repeticiones. La unidad experimental estuvo constituida por cuatro surcos de $5 \mathrm{~m}$ de longitud con una distancia entresurcos de $0.8 \mathrm{~m}$ y de $0.25 \mathrm{~m}$ entre plantas y como parcela útil se tomaron en cuenta los dos surcos centrarles, generando una densidad poblacional de 50,000 plantas $_{\text {ha-1 }}$.

\section{Variables evaluadas}

Al momento de la cosecha, se colectaron seis mazorcas de la parcela útil de cada unidad experimental, esto para cada ambiente. Se analizaron cuatro variables de respuesta relacionadas directamente con el rendimiento; el número de granos por mazorca (GM) se expresó multiplicando el número de hileras por el número de granos de cada mazorca; el número de hileras por mazorca (HM) estuvo determinado por la cantidad de granos existentes desde la base hasta la hilera más larga de cada mazorca; el peso de 100 granos (P100G) se analizó, tomando al azar una muestra representativa del peso total del grano; para el rendimiento de grano (RG) se determinó el peso total de grano ajustado al 15\% de humedad y por el número de plantas cosechadas de cada parcela útil, expresándose en $\mathrm{t} \mathrm{ha}^{-1}$.

\section{Análisis estadístico}

Los datos generados durante la medición de las variables de las 28 poblaciones, se procesaron mediante análisis de varianza combinado y por una comparación de medias mediante el procedimiento de Diferencia Mínima Significativa $\left(\mathrm{DMS}_{0.01}\right)$. El efecto de la Heterosis promedio $(\mathrm{Hp})$ se calculó de manera porcentual con la formula $\mathrm{Hp}=\left(\mathrm{F}_{1}-\mathrm{p}\right) / \mathrm{p} \times 100$; donde $\mathrm{Hp}=$ Heterosis promedio (\%); $\mathrm{F}_{1}=$ media fenotípica de la $\mathrm{F}_{1} ; \mathrm{p}=$ Comportamiento promedio de ambos progenitores. El análisis de varianza para calcular los efectos de Heterosis varietal $\left(h_{\mathrm{j}}\right)$ y específica $\left(s_{\mathrm{iij}}\right)$ de los siete progenitores y sus 21 cruzas directas (28 poblaciones), se realizó de acuerdo al método propuesto por Gardner y Eberhart (1966), bajo el modelo siguiente:

$$
Y_{j j^{\prime}}=M_{v}+1 / 2\left(V_{j}+V_{j j^{\prime}}\right)+h_{j j^{\prime}}
$$

Dónde:

$\mathrm{Y}_{\mathrm{iU}}=$ efectos genéticos de las poblaciones

$\mathrm{M}_{\mathrm{v}}=$ efecto promedio de las poblaciones progenitoras.

$\mathrm{V}_{\mathrm{j}}=$ efecto poblacional de la j-ésima población. $\mathrm{h}_{\mathrm{ij}}=$ efecto heterótico de la cruza entre la población j y la j'.

Adicionalmente el modelo subdivide al efecto heterótico en:

$h_{i j j^{\prime}}=h+h_{j}+h_{j j^{\prime}}+s_{i j}$

Dónde:

$\mathrm{h}_{\mathrm{ij}}=$ efecto heterótico

$\mathrm{h}=$ efecto de la heterosis promedio.

$h_{j}=$ efecto de la heterosis varietal producido por la variedad $\mathrm{j}$.

$h_{\mathrm{i}}=$ efecto de la heterosis varietal producido por la variedad $j^{\prime}$.

$\mathrm{s}_{\mathrm{ij}}=$ efecto de la heterosis específica producida por el cruzamiento entre las variedades $\mathrm{j}$ y j'.

La totalidad de los análisis estadísticos se realizaron con el uso del software Statistical Analysis System (SAS, 1999).

\section{RESULTADOS Y DISCUSIÓN}

\section{Comportamiento agronómico de los progenitores nati- vos de maíz y sus cruzas $F_{1}$}

El análisis de varianza mostró diferencias estadísticas $(P \leq 0.01)$ para las fuentes de variación de ambientes, poblaciones y la interacción poblaciones $x$ ambientes para las variables evaluadas de GM, HM, P100G y RG, (Tabla 2) en la fuente de variación, repeticiones dentro de ambientes se detectaron diferencias estadísticamente significativas $(P \leq 0.01)$. Los coeficientes de variación fluctuaron de 3.9 a $7.1 \%$, estos valores correspondieron a rendimiento y granos por mazorca, respectivamente. Las diferencias estadísticas detectadas en la fuente de poblaciones son el resultado del alto grado de variabilidad genética existente entre el germoplasma evaluado, lo que es efecto del origen geográfico distinto de las poblaciones. Resultados similares se observaron en un estudio de cruzas dialélicas entre poblaciones de maíz nativo de Yucatán, donde se determinó gran variabilidad genética en el germoplasma nativo y se estableció que dicho germoplasma expresó cierto nivel de heterosis (Dzib et al., 2011).

\section{Granos por mazorca (GM)}

El análisis de medias (Tabla 3) exhibió a las poblaciones progenitoras BCP1, Ratón, Moreño y BJM2 como las de mayor producción en granos por mazorca, con valores de $594.6,587.4,586.7$ y 560.6 respectivamente. Los resultados de la comparación entre los progenitores y sus cruzas $F_{1}$ indican la amplia diversidad genética presente en el germoplasma nativo de maíz (Ángeles et al., 2010; Escorcia et al., 2010). Lo anterior es sustentado por la Cruza- 35 (Morelos-Puebla), Cruza-24 (Morelos-Tamaulipas) y la Cruza-45 (Ratón-Puebla) con $506.3,527.7$ y $547.0 \mathrm{GM}$ respectivamente. En la totalidad de las cruzas, interviene el progenitor con mayor número de granos por mazorca y la diferencia en la producción fue significativa (Tabla 3). La variabilidad existente en el germoplasma puede deberse principalmente a factores de interacción de los genotipos y su adaptación con el ambiente (Palemón et al., 2012; González et al., 2013). Esta conducta se observó 
Tabla 2. Cuadrados medios de cuatro variables medidas en poblaciones progenitoras y sus Cruzas $\mathrm{F}_{1}$

Table 2. Mean squares of four variables measured in progenitor populations and their $F_{1}$ crosses.

\begin{tabular}{|c|c|c|c|c|c|c|}
\hline $\begin{array}{l}\mathbf{F V} \\
\text { GL }\end{array}$ & $\begin{array}{c}\text { Amb } \\
2\end{array}$ & $\begin{array}{c}\text { Rep/Amb } \\
6\end{array}$ & $\begin{array}{l}\text { Pob } \\
27\end{array}$ & $\begin{array}{c}\text { Pob } \times \text { Amb } \\
54\end{array}$ & $\begin{array}{c}\text { Error } \\
162\end{array}$ & $\begin{array}{l}\text { CV } \\
\text { (\%) }\end{array}$ \\
\hline $\mathrm{RG}\left(\mathrm{t} \mathrm{ha}^{-1}\right)$ & $1.09^{* *}$ & $0.03 \mathrm{~ns}$ & $0.27^{* *}$ & $0.15^{* *}$ & 0.04 & 3.9 \\
\hline GM (No.) & $29090.2^{* *}$ & 2129.4ns & $16070.3^{* *}$ & $10400.0^{* *}$ & 1648.6 & 7.1 \\
\hline HM (No.) & $6.7^{* *}$ & $0.3 \mathrm{~ns}$ & $8.0^{* *}$ & $3.9^{* *}$ & 0.8 & 7.0 \\
\hline P100G (g) & $615.2^{* *}$ & $18.7 \mathrm{~ns}$ & $45.2^{* *}$ & $29.1^{* *}$ & 10.8 & 6.6 \\
\hline $\mathrm{AP}(\mathrm{cm})$ & $143436.0^{* *}$ & $76.8 \mathrm{~ns}$ & $301.3^{* *}$ & $395.1^{* *}$ & 82.4 & 3.2 \\
\hline $\mathrm{FM}(\mathrm{d})$ & $3001.9^{* *}$ & $17.1^{* *}$ & $39.6^{* *}$ & $26.4^{* *}$ & 2.6 & 2.4 \\
\hline
\end{tabular}

$\mathrm{FV}=$ Fuentes de variación; $\mathrm{GL}=$ Grados de libertad; $\mathrm{Amb}=$ Ambientes; Rep $/ \mathrm{Amb}=$ Repeticiones dentro de ambientes; Pob = Poblaciones; Pob $\times A m b=$ Interacción genotipo $\times$ ambiente; $C V=C o-$ eficiente de variación; $\mathrm{GM}=$ Granos por mazorca; $\mathrm{HM}=$ Hileras por mazorca; $\mathrm{P} 100 \mathrm{G}=$ Peso de 100 granos; $\mathrm{RG}=$ Rendimiento de grano; $\mathrm{ns}=$ No significativo; ${ }^{*}=$ Significativo a $5 \%$; ${ }^{* *}=$ Significativo a $1 \%$.

en la Cruza-15 en donde los progenitores que intervienen (Caballeros y BCP1) con 519.6 y $594.6 \mathrm{GM}$, se colectaron originalmente en Tamaulipas (320 msnm) y Puebla (698 msnm) Tabla 1. Estos progenitores y su Cruza-15 (605.11) GM, al ser evaluadas en Ayala y Tepalcingo, Morelos (1300 y 118 msnm) presentaron una mayor adaptación, además la cruza superó al progenitor más productivo.

\section{Hileras por mazorca (HM)}

En esta variable, BCP1 (14.7), Moreño (14.7) y Ratón (13.9) obtuvieron en la mazorca mayor número de hileras, la media fue de 13.0 siendo CB029 el que presentó menor número de HM (Tabla 3). Los resultados obtenidos permiten inferir que las poblaciones progenitoras de maíz per se presentan un alto número de combinaciones alélicas, lo que es reflejo de la amplia variabilidad genética de este germoplasma, por lo que las cruzas $F_{1}$ tendrán mayor posibilidad de expresar efectos genéticos con potencial de heterosis (González et al., 2016). Este efecto puede estar presente en por lo menos cinco de la totalidad de las cruzas, siendo la Cruza-16 (14.9), Cruza-17 (14.7), Cruza-13 (14.7), Cruza-56 (14.4) y Cruza-12 (14.3) las de mayor impacto en HM, la Cruza-26 mostró el valor más bajo con 12.3 hileras. Los resultados demuestran que al menos una cruza superó o igualó en promedio el número de hileras de por lo menos el progenitor más alto. En una investigación sobre la varianza genética en maíz nativo se muestran resultados similares, además de indicar principalmente que los efectos de interacción entre alelos y de dominancia se expresan por medio de la heterosis (Muñoz et al., 2017).

\section{Peso de 100 granos (P100G)}

El análisis de medias, detectó los valores más representativos con relación al peso del grano (Cuadro 3) los progenitores de maíz nativo CB029 y Caballeros con 54.1 y 51.3 $\mathrm{g}$ fueron las más sobresalientes, mientras que en un sentido adverso el progenitor Ratón, exhibió el promedio más bajo en el peso de grano ( $48 \mathrm{~g}$ ). Con base en el comportamiento de las cruzas $F_{1}$ (Tabla 3), determinado por el análisis de medias, se mencionan cuatro cruzas como las de mayor im- pacto en el peso del grano, la Cruza-17, Cruza-12, Cruza-27, y Cruza-67 con valores correspondientes de $54.7 \mathrm{~g}, 53.2$ g,52.7 g, 52.3g respectivamente. El rendimiento del maíz, se encuentra determinado parcialmente por el peso del grano y este tiene gran impacto sobre el rendimiento final. El grado de heterosis puede ser calculado de manera porcentual con base en el comportamiento promedio de los progenitores, su comportamiento está relacionado con la adición e interacción de un gran número de factores genéticos aportados por los progenitores, estos a su vez contribuyen a la formación de cruzas inter-poblacionales con sobresaliente potencial heterótico en los componentes de rendimiento (De la Cruz et al., 2010; González et al., 2016).

\section{Rendimiento de grano (RG)}

Respecto al rendimiento como principal componente de interés, los resultados del análisis de medias (Tabla 3) muestran a las poblaciones progenitoras más sobresalientes. La media en rendimiento fue de 5.54 a $5.07 \mathrm{t} \mathrm{ha-}{ }^{-1}$, los progenitores CB029 (5.5), BCP1 (5.4), BJM2 (5.4) y BJM1 (5.3) t ha-1, fueron los más productivos en el rendimiento de grano, el progenitor que presentó el menor rendimiento fue Ratón con 5.0 tha ${ }^{-1}$. El comportamiento agronómico de las poblaciones progenitoras puede atribuirse a su diversa capacidad de adaptación al ambiente y a la amplia variación genética per se que posee este germoplasma (Nájera et al., 2010). El sobresaliente desempeño y adaptabilidad se debe en parte, a que CB029 presenta rasgos afines a la raza Tabloncillo, BJM1 y BJM2 afín a la raza Tuxpeño Norteño, ambas razas se han distinguido por su amplia distribución en el norte-centro del país y a su adaptación en diversos ambientes, además de mostrar características agronómicas sobresalientes y alta capacidad de producción (Dzib et al., 2011). En relación a la productividad que presentó el progenitor BCP1, esta puede estar relacionada a una mejor adaptación al tipo de clima y a la altitud donde se evaluó (Ayala y Tepalcingo, Morelos) ambas localidades presentan clima Cálido-subhúmedo y altitudes de (1300 y $118 \mathrm{msnm}$. La colecta de origen de este germoplasma se realizó en Coacalco, Puebla y presenta un clima Trópico-seco con altitudes de 689 msnm (Tabla 1). 
Tabla 3. Comportamiento agronómico de progenitores nativos de maíz y cruzas $F_{1}$ con relación a las características evaluadas.

Table 3. Agronomic behavior of native maize progenitors and $F_{1}$ crosses in relation to the characteristics evaluated.

\begin{tabular}{|c|c|c|c|c|}
\hline \multirow{2}{*}{$\begin{array}{l}\text { Progenitores } \\
\text { y Cruzas } F_{1}\end{array}$} & GM & HM & P100G & RG \\
\hline & (No.) & (No.) & (g) & $\left(t \cdot h a^{-1}\right)$ \\
\hline CB029 & $482.9 \mathrm{~b}$ & $11.6 \mathrm{c}$ & $54.1 \mathrm{a}$ & $5.5 \mathrm{a}$ \\
\hline BCP1 & $594.6 \mathrm{a}$ & $14.7 \mathrm{a}$ & $50.7 \mathrm{~b}$ & $5.4 \mathrm{a}$ \\
\hline BJM2 & $560.6 \mathrm{a}$ & $12.9 \mathrm{~b}$ & $51.0 \mathrm{~b}$ & $5.4 \mathrm{a}$ \\
\hline BJM1 & $556.1 \mathrm{~b}$ & $12.9 \mathrm{~b}$ & $50.3 \mathrm{~b}$ & $5.3 \mathrm{a}$ \\
\hline Moreño & $586.7 \mathrm{a}$ & $14.7 \mathrm{a}$ & $50.3 \mathrm{~b}$ & $5.1 \mathrm{~b}$ \\
\hline Caballeros & $519.6 \mathrm{~b}$ & $12.2 \mathrm{~b}$ & $51.3 \mathrm{a}$ & $5.2 \mathrm{~b}$ \\
\hline Ratón & $587.4 \mathrm{a}$ & $13.9 a$ & $48.0 \mathrm{~b}$ & $5.0 \mathrm{c}$ \\
\hline Promedio & 555.4 & 13.0 & 50.0 & 5.3 \\
\hline \multicolumn{5}{|l|}{ Cruzas F1 } \\
\hline Cruza-17 & 659.33 a & $14.68 \mathrm{a}$ & $54.78 \mathrm{a}$ & $5.50 a$ \\
\hline Cruza-37 & $585.67 \mathrm{~b}$ & $13.46 \mathrm{~b}$ & $51.56 \mathrm{~b}$ & $5.42 \mathrm{~b}$ \\
\hline Cruza-35 & $506.33 d$ & $12.37 \mathrm{c}$ & $49.00 \mathrm{~b}$ & $5.25 \mathrm{~b}$ \\
\hline Cruza-12 & $600.89 \mathrm{~b}$ & $14.31 \mathrm{a}$ & $53.22 \mathrm{a}$ & $5.45 a$ \\
\hline Cruza-57 & $558.22 \mathrm{c}$ & $13.55 \mathrm{~b}$ & $47.78 c$ & $5.10 \mathrm{c}$ \\
\hline Cruza-34 & $562.33 c$ & $13.48 \mathrm{~b}$ & $49.78 \mathrm{~b}$ & $5.02 \mathrm{c}$ \\
\hline Cruza-47 & 597.44 b & $14.22 \mathrm{a}$ & $48.44 \mathrm{c}$ & $5.06 \mathrm{c}$ \\
\hline Cruza-13 & $592.67 \mathrm{~b}$ & $14.73 a$ & $51.11 \mathrm{~b}$ & $5.47 \mathrm{a}$ \\
\hline Cruza-14 & 629.56 a & $13.93 \mathrm{~b}$ & $50.56 \mathrm{~b}$ & $5.33 \mathrm{~b}$ \\
\hline Cruza-36 & $508.44 d$ & $11.18 d$ & $48.67 \mathrm{~b}$ & $5.27 \mathrm{~b}$ \\
\hline Cruza-56 & $595.67 \mathrm{~b}$ & $14.44 \mathrm{a}$ & $49.33 \mathrm{~b}$ & $5.27 \mathrm{~b}$ \\
\hline Cruza-67 & $570.33 c$ & $13.20 \mathrm{~b}$ & $52.33 \mathrm{a}$ & $5.04 \mathrm{c}$ \\
\hline Cruza-27 & $524.33 d$ & $13.40 \mathrm{~b}$ & $52.78 a$ & $5.51 \mathrm{a}$ \\
\hline Cruza-15 & $605.11 \mathrm{~b}$ & $14.07 \mathrm{a}$ & $48.89 \mathrm{~b}$ & $5.05 \mathrm{C}$ \\
\hline Cruza-24 & $527.78 d$ & $12.87 \mathrm{c}$ & $46.67 \mathrm{c}$ & $5.23 \mathrm{~b}$ \\
\hline Cruza-45 & $547.00 \mathrm{c}$ & $12.88 \mathrm{c}$ & $47.00 \mathrm{c}$ & $5.12 c$ \\
\hline Cruza-46 & $545.33 c$ & $13.67 \mathrm{~b}$ & $45.89 c$ & $5.40 \mathrm{~b}$ \\
\hline Cruza-23 & $535.11 \mathrm{c}$ & $13.41 \mathrm{~b}$ & $47.89 c$ & $5.41 \mathrm{~b}$ \\
\hline Cruza-26 & $494.56 \mathrm{~d}$ & $12.31 \mathrm{c}$ & $48.33 c$ & $5.62 \mathrm{a}$ \\
\hline Cruza-25 & $573.11 \mathrm{~b}$ & $13.11 \mathrm{~b}$ & $47.78 c$ & $5.38 \mathrm{~b}$ \\
\hline Cruza-16 & $608.78 \mathrm{~b}$ & $14.88 \mathrm{a}$ & $49.33 \mathrm{~b}$ & $5.20 \mathrm{c}$ \\
\hline Promedio & 568.0 & 14.0 & 50.1 & 5.4 \\
\hline DMS (0.01) & 37.8 & 0.9 & 3.1 & 0.1 \\
\hline
\end{tabular}

El número de cruza codifica por el número de identificación de cada progenitor: Caballeros $=1 ; \mathrm{BJM} 1=2 ; \mathrm{BJM} 2=3$; Ratón $=4 ; \mathrm{BCP} 1=5 ; \mathrm{CB} 029=6$; Moreño $=7 ; \mathrm{GM}=$ Granos por mazorca; $\mathrm{HM}=$ Hileras por mazorca; $\mathrm{P} 100 \mathrm{G}=$ Peso de 100 granos; $R G$ = Rendimiento de grano; Cruzas con distinta letra son estadísticamente diferentes (DMS, $\mathrm{P} \leq 0.05$ ).
En una investigación sobre variabilidad morfológica en zarzamora, concluyen que los factores climáticos relacionados con la temperatura y precipitación son determinantes en la expresión de los caracteres morfológicos, así mismo en maíz se evaluaron diferentes condiciones agroclimáticas para determinar su efecto en el cultivo (Rodriguez et al., 2019; Varas y Herrera, 2019). Lo anterior menciona la importancia y la gran diversidad morfológica y genética que existe no solo en maíz sino en muchas otras especies de importancia agronómica, así mismo esta variabilidad se ve asociada principalmente a factores ambientales como lo es el clima, temperatura, precipitación altitud y latitud principalmente, está a su vez impacta en los caracteres de interés. En maíz nativo se ha demostrado que al presentar este germoplasma amplia base genética y alta capacidad de adaptación al ambiente, este puede utilizarse con fines de mejoramiento genético, eligiendo a las poblaciones progenitoras nativas de maíz con mejor comportamiento heterótico (Lopez et al., 2009).

Con relación al comportamiento de las cruzas $\mathrm{F}_{1}$ (Tabla 3), se distinguen cinco cruzas como las de mayor impacto, siendo la Cruza-26, Cruza-27, Cruza-17, Cruza-13 y Cruza-12, las más sobresalientes, la totalidad de estas superó el RG promedio de los progenitores nativos de maíz, que fue de $5.3 \mathrm{t}$ $\mathrm{ha}^{-1}$ (Tabla 3). Los resultados obtenidos en las cruzas $\mathrm{F}_{\text {, }}$ pueden estar asociados al origen geográfico distante asociado con la diversidad genética que presentan per se los progenitores nativos de maíz. Lo anterior se ve reflejado en tres de las cruzas antes mencionadas, como ejemplo, la Cruza-27 incluye progenitores con origen en Jonacatepec, Morelos y Padilla, Tamaulipas y presentan climas subhúmedos o secos y altitudes contrastantes (1290 y 230 msnm). Por lo tanto, la diversidad genética resulta ser una condición necesaria que puede en algún momento asegurar la presencia de heterosis en las cruzas (Esquivel et al., 2009), sin embargo, esta variabilidad no es suficiente para asegurar que los efectos en la heterosis sean positivos ya que estos son atribuidos principalmente a efectos aditivos, a la presencia de efectos de dominancia y sobredominancia, presencia de efectos epistáticos y a la interacción genotipo ambiente (Cress, 1966). Diversas investigaciones señalan que es necesario comprobar la heterosis, analizando el comportamiento de ambos progenitores y determinar los efectos de Heterosis varietal $\left(h_{i}\right)$ y especifica (s.i.) con el análisis de ACG y ACE (Sprague y Tatum, 1942; González et al., 2016).

\section{Expresión de la Heterosis promedio en $\mathbf{2 1}$ cruzas de maíz nativo}

Los resultados de la Heterosis promedio (Hp), expresado en porcentaje (Tabla 4), muestran a las cruzas $F_{1}$ con mayor efecto en el rendimiento de grano (GM, HM, P100G y RG). Al igual que en el análisis de medias, estas variables continúan como las de mayor importancia en la expresión de Hp. 


\section{Granos por mazorca (GM)}

Para la variable de GM, siete cruzas incrementaron el número de granos por mazorca, con porcentajes superiores al $11 \%$, siendo la Cruza-16 (21.35), Cruza-17 (19.6), Cruza-14 (13.74), Cruza-12 (11.9), Cruza-56 (10.38), Cruza-13 (9.82) y Cruza-15 (8.62) las más destacadas, la totalidad de las cruzas exhibieron efecto negativo en esta variable (Tabla 4) y en donde la Cruza-35 mostró un - $12.2 \%$ de disminución en GM. Los resultados demuestran que para la obtención de cruzas inter-poblacionales de alto rendimiento este depende del cruzamiento de los progenitores (De la Cruz et al., 2010); el haber obtenido resultados negativos, se debe en parte a la insuficiencia de diversidad genética entre las poblaciones progenitoras o bien a la presencia de efectos interalélicos o intraalélicos que reducen la expresión del carácter medio (Han et al., 1991).

\section{Hileras por mazorca (HM)}

El análisis porcentual muestra los efectos positivos de la $\mathrm{Hp}$ más sobresalientes en cuanto al número de hileras por mazorca (Tabla 4) y en donde seis de las 21 cruzas $F_{1}$ de maíz nativo mostraron porcentajes positivos de heterosis,

Tabla 4. Heterosis promedio en las 21 cruzas de maíz nativo.

Table 4. Average heterosis in the 21 crosses of native maize.

\begin{tabular}{|c|c|c|c|c|}
\hline Cruzas & $\begin{array}{c}\text { GM } \\
\text { (No.) }\end{array}$ & $\begin{array}{l}\text { HM } \\
\text { (No.) }\end{array}$ & $\begin{array}{c}\text { P100G } \\
\text { (g) }\end{array}$ & $\begin{array}{c}\text { RG } \\
\left(t \text { ha- }^{-1}\right)\end{array}$ \\
\hline \multicolumn{5}{|c|}{ Hp (\%) } \\
\hline Cruza-12 & 11.91 & 15.94 & 4.70 & 2.44 \\
\hline Cruza-13 & 9.82 & 16.93 & -0.09 & 2.62 \\
\hline Cruza-14 & 13.74 & 6.64 & 1.81 & 2.88 \\
\hline Cruza-15 & 8.62 & 5.94 & -4.13 & -5.71 \\
\hline Cruza-16 & 21.35 & 24.96 & -6.43 & -3.88 \\
\hline Cruza-17 & 19.16 & 11.81 & 7.77 & 5.36 \\
\hline Cruza-23 & -4.12 & 5.28 & -5.46 & 0.74 \\
\hline Cruza-24 & -7.7 & -2.48 & -0.05 & 0.38 \\
\hline Cruza-25 & -0.34 & -2.36 & -0.05 & -0.37 \\
\hline Cruza-26 & -4.81 & 2.03 & -7.74 & 3.11 \\
\hline Cruza-27 & -8.23 & 0.92 & 4.86 & 4.95 \\
\hline Cruza-34 & -1.91 & 0.25 & 0.56 & -3.83 \\
\hline Cruza-35 & -12.2 & -18.21 & -2.01 & -3.85 \\
\hline Cruza-36 & -0.02 & 0.59 & -7.38 & -2.95 \\
\hline Cruza-37 & 2.09 & -0.45 & 1.77 & 3.04 \\
\hline Cruza-45 & -7.28 & -11.27 & -4.72 & -2.47 \\
\hline Cruza-46 & 2.05 & 7.12 & -10.1 & 1.88 \\
\hline Cruza-47 & 1.87 & 1.63 & -1.46 & -0.97 \\
\hline Cruza-56 & 10.38 & 11.16 & -5.88 & -4.00 \\
\hline Cruza-57 & -5.42 & -4.65 & -4.82 & -3.59 \\
\hline Cruza-67 & 6.74 & 2.77 & 0.21 & -5.61 \\
\hline
\end{tabular}

$\mathrm{GM}=$ Granos por mazorca; HM = Hileras por mazorca; P100G = Peso de 100 granos; RG = Rendimiento de grano; $\mathrm{Hp}(\%)=$ porcentaje de heterosis promedio. siendo la Cruza-16 la de mayor impacto con $24.96 \%$ en el incremento de las hileras en la mazorca, esta cruza además obtuvo valores altos en HM con 14.9 y superó al promedio de ambos progenitores Caballeros (12.2 HM) y CB029 (11.6 H). Por otra parte, la Cruza-35 y Cruza-45 generó disminución en $\mathrm{HM}$ con valores de -18.21 y $-11.27 \%$ de $\mathrm{Hp}$. Los resultados obtenidos comparten cierta similitud con lo obtenido en GM, lo cual puede explicarse debido a la correlación existente en estas variables, conjuntamente puede inferirse que entre mayor sea el número de granos mayor será el número de hileras (Romero et al., 2002).

\section{Peso de 100 granos (P100G)}

Aun cuando es una variable directamente relacionada con el rendimiento y de haber obtenido progenitores y cruzas inter-poblaciones con sobresaliente peso en el grano (Tabla $3)$, esta variable fue la de menor impacto en el efecto de $\mathrm{Hp}$, solo tres cruzas presentaron valores positivos en el peso del grano en gramos (Tabla 4). La Cruza-17, Cruza-27 y Cruza-12 presentaron porcentajes de 7.77, 4.86 y 4.70 respectivamente, la totalidad de las cruzas $F_{1}$ exhibió disminución en el peso de 100 granos. Los resultados muestran que aun cuando el peso del grano está relacionado al rendimiento, la expresión de la heterosis puede no presentarse en la mayoría de las poblaciones nativas de maíz, esto puede estar relacionado a diversos factores, principalmente aquellos que inhiben la expresión alélica (Han et al., 1991; De la Cruz et al., 2010).

\section{Rendimiento de grano (RG)}

Con relación al efecto expresado en porcentaje (\%) del análisis de Heterosis promedio (Hp) [Tabla 4], nueve de la totalidad de las cruzas presentaron valores positivos que van de $5.37 \%$ (Cruza-17) a 0.38\% (Cruza-24), la Cruza-15 presentó efecto de heterosis negativo con $-5.71 \%$ en la disminución del rendimiento. Los resultados presentados en la $\mathrm{Hp}$, son atribuibles a la acción conjunta de factores genéticos no aditivos, principalmente efectos de dominancia (Muñoz et al., 2017), los cuales determinaron un mayor efecto en las cruzas $F_{1}$ y por lo tanto una mayor expresión en el rendimiento. Este comportamiento heterótico puede apreciarse en la Cruza-17, Cruza-27, Cruza-26, Cruza-13 y Cruza-12 ya que estas presentaron los valores más altos en el rendimiento de grano (Tabla 3). Sin embargo 11 de las 21 cruzas exhibieron valores negativos. Este comportamiento, muestra que el valor promedio de las cruzas determinado por el análisis de medias no siempre es un indicador del valor porcentual de la heterosis; es decir valores altos de heterosis no siempre corresponden a valores altos de rendimiento (Gaytán y Meyec, 2010). Los resultados concuerdan con investigaciones sobre heterosis en rendimiento de grano de cruzas simples de maíz (Reyes et al., 2004).

\section{Estimación de los efectos de Heterosis varietal $\left(h_{j}\right)$ de los progenitores de maíz nativo \\ Al someter las cuatro variables al análisis de varianza por el método de Gardner y Eberhart, solo las variables de}


GM y RG, presentaron diferencias estadísticas altamente significativas. Los resultados presentados en el Tabla 5, muestran los efectos genéticos de heterosis que influyeron en la expresión de los 28 genotipos evaluados. Se observaron diferencias estadísticas altamente significativas $(P \leq 0.01)$ para los genotipos en las variables evaluadas, posteriormente al descomponer la fuente de variación de genotipos en Progenitores y Cruzas, se observaron también diferencias estadísticas altamente significativas $(P \leq 0.01)$ en las variables analizadas, subsecuentemente al dividir la fuente de variación de Cruzas en Heterosis promedio (h), Heterosis varietal $\left(h_{\mathrm{j}}\right)$ y Heterosis específica $\left(\mathrm{s}_{\mathrm{j} j}\right)$, se observaron diferencias estadísticas altamente significativas $(P \leq 0.01)$ en granos por mazorca y en el rendimiento de grano, solo ese observaron diferencias altamente significativas $(P \leq 0.01)$ en Heterosis varietal y Heterosis promedio en Heterosis específica la diferencia fue no significativa.

Tabla 5. Cuadrados medios para las variables de granos por mazorca y rendimiento de grano analizadas mediante el modelo de (Gardner y Eberhart, 1966).

Table 5. Mean squares for the variables of grains per ear and grain yield analyzed by the model of (Gardner and Eberhart, 1966).

\begin{tabular}{lccc}
\hline FV & GL & $\begin{array}{c}\text { GM } \\
\text { (No.) }\end{array}$ & $\begin{array}{c}\text { RG } \\
\text { (t ha-1) }\end{array}$ \\
\hline Poblaciones & 27 & $16070.3^{* *}$ & $0.27^{* *}$ \\
Progenitores & 6 & $15161.1^{* *}$ & $0.24^{* *}$ \\
Cruzas & 21 & $16330.1^{* *}$ & $0.28^{* *}$ \\
$\quad$ Heterosis promedio & 1 & $7505.2 \mathrm{~ns}$ & $0.43^{* *}$ \\
$\quad$ Heterosis varietal & 6 & $44774.4^{* *}$ & $0.87^{* *}$ \\
$\quad$ Heterosis específica & 14 & $22040.2 \mathrm{~ns}$ & $0.11^{* *}$ \\
Error & 224 & 4016.1 & 0.08 \\
CV (\%) & & 7.1 & 3.9 \\
\hline
\end{tabular}

$\mathrm{FV}=$ Fuente de variación; $\mathrm{GL}=$ Grados de libertad; $\mathrm{CV}=$ Coeficiente de variación; $\mathrm{GM}=$ Granos por mazorca; $\mathrm{RG}=$ Rendimiento de grano; NS = No significativo; $^{*}=$ Significativo a $5 \%{ }^{* *}=$ Significativo a $1 \%$.

Los resultados encontrados en los 28 genotipos de maíz nativo, se deben a la gran variación que existe en dicho germoplasma, por lo tanto y en ese sentido los resultados mostrados en Progenitores y Cruzas se ve asociado con esa misma variabilidad. La respuesta significativa encontrada en Heterosis promedio, Heterosis varietal y Heterosis específica, permite por un lado corroborar el valor de las 28 poblaciones nativas de maíz a través de su heterosis promedio, así mismo aprovechar la estimación de la heterosis varietal y determinar los parámetros de la heterosis específica, para la formación de combinaciones germoplásmicas de alto rendimiento derivadas de cruzamientos inter-poblacionales específicos (González et al., 2016). Resultados similares se observaron en poblaciones heteróticamente contrastantes de maíz de origen tropical y subtropical, donde se determinó la magnitud de los componentes genéticos de la variación para identificar combinaciones específicas entre las cruzas e identificar germoplasma con potencial de heterosis en rendimiento de grano (Preciado et al., 2005).

\section{Granos por mazorca (GM)}

Los progenitores CB029 y BCP1 mostraron los mayores efectos de heterosis varietal $\left(h_{j}\right)$ en la producción de granos por mazorca (Tabla 6), ambos progenitores de maíz nativo aumentan respectivamente 72.37 y 39.9 en GM cuando intervienen en cualquier cruza $F_{1}$. Caso contrario con el progenitor Caballeros, el cual tiende a producir un efecto varietal negativo en el número de granos por mazorca, lo que indica que cualquier cruza $F_{1}$ en la que intervenga este progenitor disminuirá -35.71 granos en la mazorca de la cruza inter-poblacional. Los resultados pueden estar relacionados con el tipo de acción génica que presente el germoplasma estudiado y el cual se ve influenciado mayormente por presencia de la acción génica aditiva sobre los efectos no aditivos (González et al., 2016). Esto está acorde a los resultados encontrados en un estudio de heterosis y aptitud combinatoria general en genotipos tropicales de maíz, el cual concluye que para obtener mejor respuesta heterótica es conveniente combinar germoplasma proveniente de diferentes áreas de adaptación, bajo el supuesto de que a mayor divergencia genética habrá mayor heterosis y que además si el progenitor presenta efecto varietal $\left(h_{\mathrm{j}}\right)$ las cruzas o al menos una de ellas podrá obtener altos rendimientos (Esquivel et al., 2009; Preciado et al., 2005).

Tabla 6. Efecto de heterosis varietal $\left(h_{j}\right)$ de las poblaciones progenitoras nativas de maíz evaluadas.

Table 6. Varietal heterosis effect $\left(h_{j}\right)$ of the native maize progenitor populations evaluated.

\begin{tabular}{lcc}
\hline Progenitores & $\begin{array}{c}\text { GM } \\
(\text { No. })\end{array}$ & $\begin{array}{c}\text { RG } \\
\left(\mathrm{t} \mathrm{ha-}^{-}\right)\end{array}$ \\
\hline Caballeros & -35.71 & -0.02 \\
BJM2 & 0.83 & 0.04 \\
\hline BJM1 & 4.73 & 0.06 \\
Ratón & 31.73 & -0.25 \\
BCP1 & 39.33 & 0.12 \\
CB029 & 72.37 & 0.22 \\
\hline Moreño & 31.43 & -0.17 \\
\hline
\end{tabular}

$\mathrm{RG}=$ Rendimiento de grano; $\mathrm{GM}=$ Granos por mazorca.

\section{Rendimiento de grano (RG)}

Respecto al rendimiento de grano como principal componente de interés, los resultados del análisis de efectos genéticos, mostraron presencia de efecto varietal producido por las poblaciones progenitoras nativas de maíz (Tabla 6). Con relación al rendimiento de grano (RG), los progenitores que presentaron efecto de Heterosis varietal $\left(h_{j}\right)$ fueron CB029 de origen en el estado de Morelos y BCP1 con origen en el estado de Puebla, con valores de 0.22 y $0.12 \mathrm{t} \mathrm{ha}^{-1}$ respectivamente, en tanto que, en un sentido inverso los progenitores Ratón, Moreño y Caballeros con origen en el estado de Tamaulipas tuvieron efectos negativos en el RG (-0.25,$0.17,-0.02 \mathrm{t} \mathrm{ha}{ }^{-1}$, respectivamente. Con base en el modelo de Gardner y Eberhart en donde la ACG de los progenitores es un estimador de los efectos de Heterosis varietal $\left(h_{j}\right)$, los 
resultados muestran aquellos genotipos útiles por su significativo efecto varietal. Los diferentes efectos mostrados, se deben al comportamiento per se y a la constitución génica de los progenitores nativos de maíz, los valores registrados son un indicador de la variabilidad existente en los progenitores y de cómo esta variación contribuye a la predicción en el efecto de la progenie, esto permite seleccionar genotipos que combinen características superiores. Los resultados concuerdan con una investigación sobre parámetros genéticos y heterosis en poblaciones nativas de maíz (González et al., 2016).

\section{Estimación de los efectos de Heterosis específica $\left(s_{\mathrm{jj}}\right)$ en las 21 cruzas $F_{1}$ de maíz nativo}

En cuanto a los efectos de heterosis específica $\left(s_{\mathrm{jij}}\right)$ en las cruzas $F_{1}$ de maíz nativo consideradas en esta investigación, se muestran los resultados con respecto a las dos variables de mayor impacto en dicho efecto (Tabla 7).

\section{Granos por mazorca (GM) y Rendimiento de grano (RG)}

En general se observaron efectos de Heterosis específica ( $\mathrm{s}_{\mathrm{ij}}$ ) positivos para GM y RG (Tabla 7 ). LaCruza-46 exhibió el valor positivo más alto en $\mathrm{RG}\left(0.21 \mathrm{t} \mathrm{ha}^{-1}\right)$ pero en GM mostró un valor negativo (-5.98), siendo ésta la que tuvo el mayor efecto $\left(\mathrm{s}_{\mathrm{jj}}\right)$ en el rendimiento de grano, la Cruza-17, obtuvo valores positivos en GM (16.20) y RG $\left(0.17 \mathrm{t} \mathrm{ha}^{-1}\right)$. Otra Cruza que presentó un efecto importante fue la Cruza-56 la cual expresó valores positivos en el número de granos por mazorca y rendimiento de grano, en contraste la Cruza-57 exhibió valores negativos en las dos variables relacionadas al rendimiento (GM y RG). Esto indica que en la herencia de las cruzas intervinieron genes aditivos y no aditivos los cuales permitieron la expresión de características favorables y no favorables (Ramírez et al., 2004).

Los valores altos encontrados en el análisis de Heterosis específica pueden deberse a la suma de efectos aditivos de los genes de los progenitores nativos de maíz, como es el caso de la Cruza-56 donde los progenitores que intervienen mostraron altos rendimientos ( 5.5 y $5.4 \mathrm{t} \mathrm{ha}^{-1}$ ) o bien, a los efectos de la interacción de los alelos dominantes de un progenitor con los alelos recesivos de otro progenitor (Cruza-46), en este caso intervinieron los progenitores con el más alto y el más bajo rendimiento ( 5.5 y $5.1 \mathrm{t} \mathrm{ha}^{-1}$ ). Por otra parte, se observó que cuando los progenitores provienen del mismo origen pueden diferir en el comportamiento de Heterosis varietal y específica (Vasal et al., 1995). Los progenitores Ratón, Caballeros y Moreño tienen parentesco en su origen (Tamaulipas) pero presentan diferentes efectos de $\left(s_{\mathrm{jij}}\right)$ cuando se cruzan con el progenitor CB029 (Morelos), mientras que la Cruza-46 presenta un valor positivo $\left(0.21 \mathrm{t} \mathrm{ha}^{-1}\right)$, la Cruza-16 y Cruza-67 muestran valores negativos de -0.16 y -0.24 (t ha$\left.{ }^{1}\right)$, siendo éste el valor más bajo de Heterosis especifica. La evaluación de los efectos genéticos, tanto varietales como específicos mediante cruzamientos dialélicos es eficiente en la clasificación de progenitores e identifica fuentes de germoplasma útiles en programas de mejoramiento genético
Tabla 7. Efecto promedio de la Heterosis específica $\left(\mathrm{S}_{\mathrm{jj}}\right)$ en las 21 cruzas de maíz nativo.

Table 7. Average effect of the specific heterosis $\left(\mathrm{S}_{\mathrm{jij}}\right)$ in the 21 crosses of native maize.

\begin{tabular}{lcc}
\hline Cruzas $\mathbf{F}_{1}$ & $\begin{array}{c}\text { GM } \\
\text { (No.) }\end{array}$ & $\begin{array}{c}\text { RG } \\
\text { (t ha- }\end{array}$ \\
\hline Cruza-12 & 5.67 & -0.07 \\
\hline Cruza-13 & -9.51 & 0.11 \\
\hline Cruza-14 & 3.60 & 0.10 \\
\hline Cruza-15 & -16.05 & -0.08 \\
\hline Cruza-16 & 0.09 & -0.16 \\
\hline Cruza-17 & 16.20 & 0.17 \\
\hline Cruza-23 & 21.04 & -0.08 \\
\hline Cruza-24 & -10.06 & -0.12 \\
\hline Cruza-25 & 40.07 & 0.03 \\
\hline Cruza-26 & -26.02 & 0.15 \\
\hline Cruza-27 & -30.69 & 0.07 \\
\hline Cruza-34 & 17.53 & -0.18 \\
\hline Cruza-35 & -33.67 & 0.05 \\
\hline Cruza-36 & -19.09 & -0.06 \\
\hline Cruza-37 & 23.70 & 0.13 \\
\hline Cruza-45 & -16.78 & 0.06 \\
\hline Cruza-46 & -5.98 & -0.06 \\
\hline Cruza-47 & 11.69 & -0.24 \\
\hline Cruza-56 & 49.16 & -0.10 \\
\hline Cruza-57 & -22.73 & 0.08 \\
\hline Cruza-67 & 1.84 & \\
\hline & & 0.21 \\
\hline
\end{tabular}

El número de cruza está en función del código de cada progenitor: Caballeros $=1 ; B J M 1=2 ; B J M 2=3 ;$ Ratón = 4; $B C P 1=5 ; C B 029=6 ;$ Moreño = 7; AP $=$ Altura de planta; $\mathrm{GM}=$ Granos por mazorca; $\mathrm{P100G}=$ Peso de 100 granos; $\mathrm{RG}=$ Rendimiento de grano.

(Gaytán y Meyec, 2010).

\section{CONCLUSIONES}

Las poblaciones nativas de maíz mostraron un alto grado de variabilidad genética inter-poblacional en todas las variables evaluadas. El distinto origen genético de los progenitores nativos de maíz, así como la interacción genotipo por ambiente aseguraron la expresión de heterosis en las cruzas $F_{1}$. La Heterosis promedio se manifestó de manera importante particularmente, en la Cruza-17 y Cruza-26, mostrando porcentajes de heterosis positiva para granos por mazorca y rendimiento de grano. Los diferentes efectos varietales manifestados se deben al comportamiento per se y a la constitución génica de los progenitores nativos de maíz, en ese sentido los progenitores que mostraron efectos de Heterosis varietal significativos fueron BCP1 y CB029 ambos de diferente origen (Puebla y Morelos). La Cruza-56 fue la que manifestó un impacto significativo en el efecto de Heterosis específica $\left(s_{\mathrm{jj}}\right)$ e incluye a los progenitores antes mencionados, otras cruzas de importancia fueron las Cruza-25, Cruza-37 y la Cruza-17. Los resultados obtenidos 
permiten seleccionar a las poblaciones nativas de maíz y las cruzas $F_{1}$ antes mencionadas como germoplasma base para un programa de selección recurrente y de mejoramiento inter-poblacional.

\section{REFERENCIAS}

Ángeles, G.E., Ortiz, T.E., López, P.A. y López, R.G. 2010. Caracterización y rendimiento de poblaciones de maíz nativas de Molcaxac, Puebla. Revista Fitotecnia. Mexicana. 33 (4): 287-296.

Cress, C.E. 1966. Heterosis of the hybrid related to gene frequency differences between two populations. Genetics. 53: 269-274.

De la Cruz, E., Castañón, N.G., Brito, M.N.P., Gómez, V.A., Robledo, T.V. y Lozano, del Río A.J. 2010. Heterosis y aptitud combinatoria de poblaciones de maíz tropical. Revista Internacional de Botánica Experimental. 79 (1): 11-17.

Dzib, S.L.A., Segura, C.J.C., Ortega, P.R. y Latournerie, M.L. 2011. Cruzas dialélicas entre poblaciones nativas de maíz de Yucatán y poblaciones mejoradas. Tropical and Subtropical Agroecosystems. 14: 119-127.

Escorcia, G.L.N., Molina, G.J.D., Castillo, G.F. y Mejía, C.J.A. 2010. Rendimiento, heterosis y depresión endogámica de cruzas simples de maíz. Revista. Fitotecnia. Mexicana. 33 (3): 271 279.

Esquivel, E.G., Castillo, G.F., Hernández, C.J.M., Santacruz, V.A., García, S.G., Acosta, G.J.A. y Ramírez, H.A. 2011. Heterosis en maíz del Altiplano de México con diferente grado de divergencia genética. Revista Mexicana de Ciencias Agrícolas. 2 (3): 331-344.

Esquivel, E.G., Castillo, G.F., Hernández, C.J.M., Santacruz, V.A., García, S.G., Acosta, G.A. y Ramírez. H.A. 2009. Aptitud combinatoria y heterosis en etapas tempranas del desarrollo del maíz. Revista Fitotecnia Mexicana. 32 (4): 311-318.

Falconer, D.S. y Mackay, T.F.C. 1996. Introduction to quantitative genetics. $4^{\text {th }}$ Edition. Longman. Essex, England. 464p.

Gardner, C.O. y Eberhart, S.A. 1966. Analysis and interpretation of the variety cross diallel and related populations. Biometrics. 22: 439-452.

Gaytán, B.R. y Mayek, P.N. 2010. Heterosis en híbridos de maíz producidos de cruzamientos entre progenitores de valles altos $\mathrm{x}$ tropicales. Investigación y ciencia de la universidad autónoma de Aguascalientes. 18 (48): 4-8.

González, C.M.E., Palacios, R.N., Espinoza, B.A. y Bedoya, S.C.A. 2013. Diversidad genética en maíces nativos mexicanos tropicales. Revista. Fitotecnia. Mexicana. 36: 329-338.

González, M.J., López, S.J.A., Estrada, D.B., Delgado, M.R., Pecina, M.J.A., Varela, F.E.S., Osorio, H.E. y Rocandio, R.M. 2016. Parámetros genéticos y heterosis en líneas derivadas de poblaciones nativas de maíz tropical de Tamaulipas. Revista Mexicana de Ciencias Agrícolas. 7 (2): 387-399.

Griffing, B.J. 1956. Concept of general and specific combining ability in relation to diallel crossing systems. Australian Journal of Biological Sciences, 9: 463-493.

Gutiérrez, R.E., Palomo, G.A., Espinoza, B.A. y de la Cruz, L.E. 2002. Aptitud combinatoria y heterosis para rendimiento de líneas de maíz en la comarca lagunera, México. Revista. Fitotecnia. Mexicana. 24 (3): 271-277.

Han, G.C., Vasal, S.K., Beck, D.L. y Elias, E. 1991.Combining ability of inbred lines derived from CIMMYT maize (Zea mays L.) germplasm. Maydica. 36: 57-67.
Hoecker, N., Keller, B., Piepho, H.P. y Hochholdinger, F. 2006. Manifestation of heterosis during early maize (Zea mays $L$.) root development. Theoretical and Applied Genetics. 112: 421-429.

Lopez, C.G., Bertoia, L.M. y Burak, R. 2009. Heterosis and heterotic patterns among maize landraces for forage. Crop Breeding and Applied Biotechnology 9 (3): 229-238.

Maldonado, F.J., Pinto, M.R.M. y Fernandes, H.J.A. 2009. Capacidade combinatória e heterose em populações de milho crioulo. Ciência Rural, Santa Maria. 39 (2): 332-339.

Moll, R.H., Salhuana, W.S. and Robinson, H.F. 1962. Heterosis and genetic diversity in variety crosses of maize. Crop Science. 2: 197-198.

Muñoz, R.L.A., Navarro, G.E., De-la-Rosa, I.M., Pérez, R.L.A. y Caamal, D.A.E. 2017. Estimación de varianzas genéticas en ocho variedades criollas de maíz para el Bajío mexicano. Agronomía Mesoamérica. 28 (2): 455-464.

Palemón, A.F., Gómez, M.N.O., Castillo, G.F., Ramírez, V.P., Molina, G.J.D. y Miranda, C.S. 2012. Potencial productivo de cruzas intervarietales de maíz en la región semicálida de Guerrero Revista Mexicana de Ciencias Agrícolas. 3 (1): 157-171.

Preciado, O.R.E., Terrón, I.A.D., Gómez, M.N.O. y Robledo, G.E.I. 2005. Componentes genéticos en poblaciones heteróticamente contrastantes de maíz de origen tropical y subtropical. Agronomía. Mesoamericana 16 (2): 145-151.

Ramírez, D.J.L., Chuela, B.M., Soltero, D.L., Franco, M.J., Morfín, V.A., Vidal, M.V.A., Vallejo, D.H.L., Caballero, H.F., Delgado, M.H., Valdivia, B.R. y Ron, P.J. 2004. Patrón heterótico de maíz amarillo para la región centro-occidente de México. Revista Fitotecnia Mexicana. 27 (1): 13-17.

Reyes, L. D., Molina, G.J.D., Oropeza, R.M.A. y Moreno, P.E. 2004. Cruzas dialélicas entre líneas autofecundadas de maíz derivadas de la raza Tuxpeño. Revista Fitotecnia Mexicana. 27 (1): 49-56.

Rodriguez, B.G., Segura, L.S.D., Cruz, I.S., López, M.J., Gutierrez, E.A., Cruz, H.N., Carrillo, S.J. y Valenzuela, N.L.M. 2019. Distribución y variabilidad morfológica de especies de zarzamoras en México (Rubus spp L.). Biotecnia. 21 (3): 7-105.

Romero, P.J., Castillo, G.F. y Ortega, P.R. 2002. Cruzas de poblaciones nativas de maíz de la raza Chalqueño: II. Grupos genéticos, divergencia genética y heterosis. Revista Fitotecnia Mexicana. 25 (1): 107-115.

Statistical Analysis System Institute (SAS). 1999. SAS user's guide. Statistics. Versión 8.2.SAS Inst. Cary, N.C.

Soengas, P., Ordás, B., Malvar, R.A., Revilla P. y Ordás A. 2003. Heterotic patterns among flint maize populations. Crop Science 43: 844-849.

Sprague, G.F. y Tatum, L.A. 1942. General vs. specific combining ability in single crosses of corn. Journal American Society Agronomy 43: 923-932.

Vasal, S.K.G., Srinivasan, N., Vergara, A. y González, C.F. 1995. Heterosis y aptitud combinatoria en germoplasma de maíz de valles altos. Revista Fitotecnia Mexicana.18: 123-139.

Vergara, A.N., Rodríguez, H.S.A. y Córdova, O.H.S. 2005. Aptitud combinatoria general y específica de líneas de maíz (Zea mays L.) tropical y subtropical. Agronomía Mesoamericana. 16 (2): 137-143.

Varas, M.C. y Herrera, S.M. 2019. Caracterización del período de crecimiento agroclimático del maíz (zea maíz) en la provincia los ríos. Biotecnia. 21 (1): 54-59. 